\title{
Sleep position, autonomic function, and arousal
}

\author{
B C Galland, G Reeves, B J Taylor, D P G Bolton
}

\begin{abstract}
Aims-To investigate and compare heart rate variability (HRV) and responses of heart rate and arousal to head-up tilting in infants sleeping prone and supine.

Methods-Thirty seven healthy infants aged 2-4 months were studied. HRV was measured for 500 beats while they were in a horizontal position. Subjects were then tilted $60^{\circ}$ head-up, and heart rate recorded over 1 minute and arousal responses observed. Data were collected during both quiet and active sleep for both prone and supine sleep positions.

Results-HRV, as assessed by the point dispersion of Poincaré plots, was significantly reduced in the prone position for both sleep states. Sleep position did not influence the changes in heart rate seen during a head-up tilt. Full awakening to the tilt was common in active sleep but significantly less so in the prone position ( $15 \%$ of prone tests $v s 54 \%$ supine). Full awakening to the tilt rarely occurred during quiet sleep in either sleep position. Conclusion-This study provides some evidence that blunted arousal responses and/or altered autonomic function are a feature of the prone sleeping position. Decreased HRV may be a sign of autonomic impairment. It is seen in many disease states and in infants who later die of sudden infant death syndrome (SIDS).

(Arch Dis Child Fetal Neonatal Ed 1998;78:F189-F194)
\end{abstract}

Keywords: heart rate variability; head-up tilting; prone position; supine position

Prone sleeping position has consistently been shown to increase the risk of sudden infant death syndrome (SIDS) and is now believed to be causally related. ${ }^{1-3}$ Many theories have been suggested for the biological mechanisms that increase the risk during prone sleeping. Airways obstruction has been implicated by several researchers, ${ }^{45}$ as have rebreathing into soft bedding ${ }^{67}$ and increasing susceptibility to hyperthermia. ${ }^{8}$ Most theories postulate that SIDS would occur only if the infant were less responsive than normal as a result of antenatal and/or postnatal factors which influence normal development.

Autonomic control in infants who die of sudden infant death syndrome (SIDS) may be immature. Epidemiological and clinical studies have shown that babies who die of SIDS or are at high risk of SIDS are more likely to have soaking sweats suggesting autonomic dysfunction. ${ }^{9}{ }^{10}$ Recent studies of autonomic function in infancy have examined heart rate dynamics using Poincaré plots; these have revealed that SIDS babies had significantly different patterns of heart rate variability (HRV). ${ }^{11}$ Studies in infants who have sustained an ALTE (apparent life threatening event) show a different pattern of heart rate control compared with that in other babies. Other studies have shown significantly reduced changes in heart rate and blood pressure in response to a head-up tilt. ${ }^{12-13}$ Some preliminary studies by White et $a l^{13}$ suggest that autonomic function was affected by sleep position.

The autonomic nervous system functions as an overall controlling system for cardiorespiratory control and arousal. Its need to operate efficiently in life threatening situations may be essential for survival.

This study aimed to investigate further autonomic function and arousal from sleep with changes in infant sleep position.

\section{Methods}

Thirty seven full term infants (19 girls, 18 boys), born at Queen Mary Maternity Unit at Dunedin Hospital between September 1995 and June 1996, were studied. The mean age of infants at the time of study was 11.4 weeks, with a range from $8-17$ weeks and mean weight $5790 \mathrm{~g}$ (range 4470 to $7270 \mathrm{~g}$ ). Postal invitations were sent to parent(s) whose medical records complied with our inclusion and exclusion criteria. Recruitment rate was $29 \%$. All babies included were born at the Unit. Exclusion criteria were: living outside the Dunedin area; severe postnatal illness; major congenital abnormality or severe illness at time of study. Informed written consent was obtained from the parent(s) of all infants. The study was approved by the Ethics Committee Otago, Southern Regional Health Authority, Dunedin, New Zealand.

The babies were brought in to Dunedin Hospital for a morning or afternoon nap in the research nursery, a quiet room with dim lighting. Room temperature was maintained at $22^{\circ} \mathrm{C}$. Setup for recordings involved placement of ECG electrodes and respibands and fitting of a harness (Jolly Jumper Inc., Cambridge, Canada) which when attached to a modified pram, supported the infants at $60^{\circ}$ during the tilt. The infants were then fed and randomly positioned prone or supine to sleep in the pram.

The respiratory pattern was recorded by inductive plethysmography (Respitrace model 150; Respitrace Co, NY, USA) and respiratory rate only was measured from this. The electrocardiogram (ECG) sensors were placed in a modified lead II position on the chest wall to give inverted $T$ waves and the signals amplified by a MacLab Bio Amp (ADInstruments Pty Ltd, Australia). The respiratory and ECG signals were relayed through the integrated 


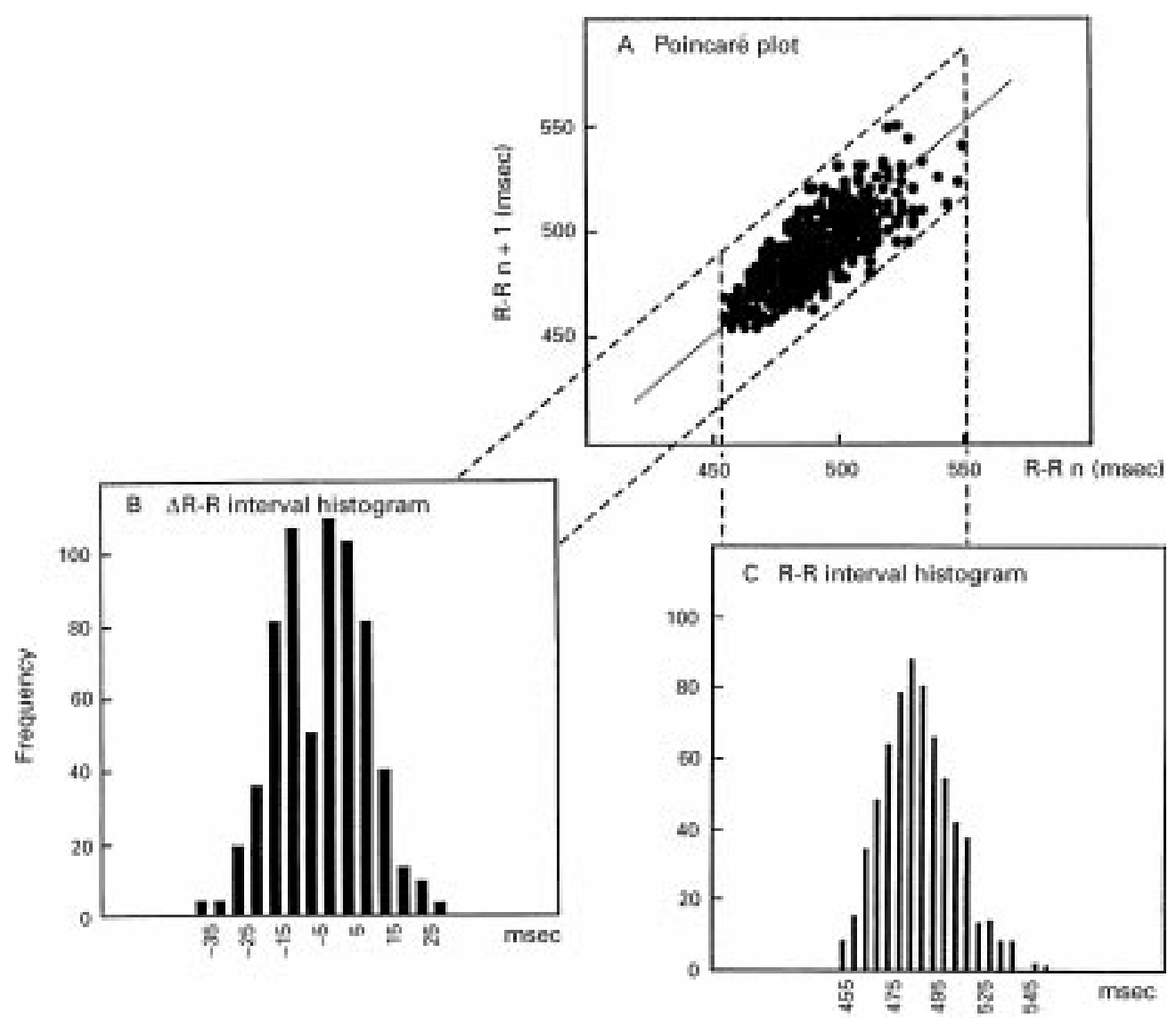

Figure 1 Poincaré plot $(A)$ showing scatter plot of $R-R$ intervals $(R-R n)$ plotted against the next intervals $(R-R . n+1)$, and giving a visual display of the overall beat to beat variability. Data are taken from one infant during quiet sleep in the prone position. Dispersal of the data are described by measuring the standard deviation of the R-R interval (SDR-R) histogram data $(C)$ and the standard deviation of the $\triangle R-R$ interval (SD $\triangle R-R$. histogram data (B). The calculated $S D R-R$ and $S D \triangle R-R$ are 16.1 and $10.9 \mathrm{~ms}$, respectively, giving an aspect ratio (SD $R-R / S D \triangle R-R$ ) of 1.48 .

hardware/software system of the MacLab (ADInstruments Pty Ltd, Australia) at a sampling frequency of $500 \mathrm{~Hz}$.

Once infants were asleep, their sleep state was ascertained by inspection of eye, mouth, and hand movements and the respiratory trace, as described by Anders et al. ${ }^{14}$ Quiet sleep was defined as a state of regular breathing with no rapid eye movements or facial movements. Active sleep contained at least some rapid eye movements, irregular breathing, and often hand or mouth movements. An ECG was recorded for 500 heart beats for measurement of HRV. Infants were then tilted head-up by pulling the pram downwards to rest on a support attached to the pram that was positioned at an angle of $60^{\circ}$ to the floor. The tilt took 2-3 seconds to complete. Heart rate responses and arousal responses to this fall in blood pressure were measured. The baby was then returned to a horizontal position and given a three minute recovery period to allow any physiological processes affected by the tilt to return to normal. The baby was turned over to the alternative sleep position and the procedures repeated. Assessments of HRV and responses to tilting were made twice in each sleep state and sleep position in over $95 \%$ of all subjects. Thirteen of the babies were studied in both sleep positions within one nap, the others $(n=24)$ were brought back for study in the other sleep position within a day or no more than a week of the initial test.

\section{MEASUREMENTS}

Heart rate variability

R-R intervals were detected over 500 consecutive beats and Poincaré plots (scatter plots of the length of each $\mathrm{R}-\mathrm{R}$ interval plotted against the length of the previous R-R interval) constructed using the heart rate variability extension of the software program ADI Chart V.3.4.2 (ADInstruments Pty Ltd, Australia). R-R data were first subjected to automated and manual correction of artefact contaminated sections. HRV was quantified from the scatter of Poincaré data (fig 1), according to Kamen and Tonkin ${ }^{15}$ as:(i) the standard deviation of the change in R-R interval from one beat to the next (SDR-R) which relates to the variance of the R-R interval histogram data projected on to the $\mathrm{x}$ axis; (ii) the standard deviation of the difference between $R-R$ intervals ( $S D \triangle R-R$ ) which relates to the variance of $R-R$ interval histogram data points parallel to the line of identity (the diagonal line at an angle of $45^{\circ}$ to both axes); (iii) the aspect ratio-ratio of variance (or $S D$ ) - of SDR-R to $S D \triangle R-R$. Points spread closely along the line of identity show little variation. Points above and below this line mean a shorter and longer R-R 


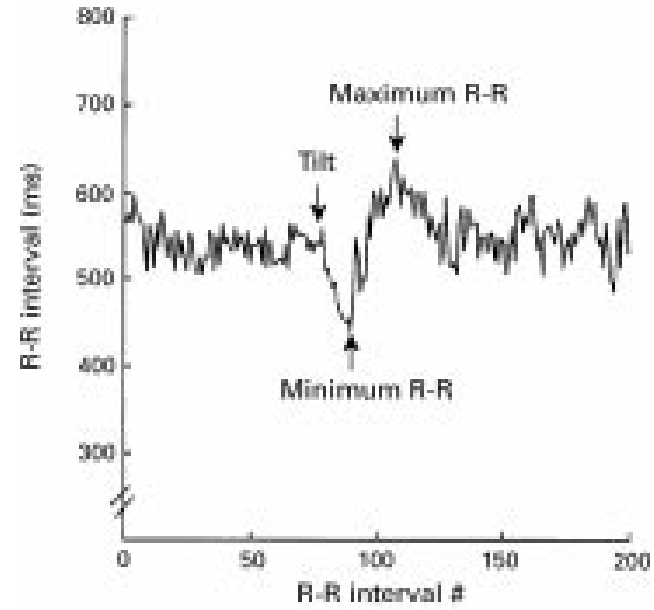

Figure 2 Example of the R-R interval changes before, during, and after a $60^{\circ}$ head-up tilt. Maximum $R-R$ is calculated as that occurring within 25 beats of the tilt.

interval, respectively, relative to the previous $\mathrm{R}-\mathrm{R}$ interval.

Heart rate response to tilt

Analysis of R-R interval changes in response to the head-up tilt could be analysed only in cases where the subjects stayed asleep during the tilt (arousal score 0). Using similar methodology to that described by Fox and Matthews, ${ }^{12}$ the heart rate response to the tilt was analysed by measuring the ratio of: the minimum $\mathrm{R}-\mathrm{R}$ length (tachycardia) seen after the tilt to the next maximum R-R length (bradycardia) within 25 beats (fig 2). In control infants (no family history of SIDS or ALTE), this ratio is greater than $1 .^{12}$

\section{Arousal response to the tilt}

Infants were watched closely during the tilt to record an arousal score based on their behavioural response to the tilt: (0) no movement at all; (0.5) startle in response to tilt but remained asleep; (1) awake with gross head movement and/or eyes open but asleep again within 15-30 seconds and remaining so on return to horizontal; (2) full awakening with eyes open and usually crying.

The SAS procedure PROC MIXED was used to compare heart rate variation, respiratory data, and R-R interval changes in response to the tilt for sleep position and sleep state. This procedure uses Restricted Estimated Maximum Likelihood analyses which means that cases where some repeated data are missing can be used. Arousal scores were compared by matched analyses using the Wilcoxon sign rank test. A p value $<0.05$ was regarded as significant.

Table 1 HRV measures during quiet and active sleep comparing prone and supine sleeping positions

\begin{tabular}{llllll}
\hline & $\begin{array}{l}\text { No of } \\
\text { subjects }\end{array}$ & $\begin{array}{l}\text { Mean }(S D) \text { heart } \\
\text { rate bpm }\end{array}$ & $\begin{array}{l}\text { Mean }(S D) \\
R-R(m s)\end{array}$ & $\begin{array}{l}\text { Mean (SD) } \\
\Delta R-R(m s)\end{array}$ & $\begin{array}{l}\text { Aspect ratio } \\
(S D)\end{array}$ \\
\hline $\begin{array}{l}\text { Quiet sleep } \\
\text { Prone }\end{array}$ & 37 & $129.4(9.3)$ & $16.30(7.1)^{\star \star}$ & $14.06(8.8)^{\star}$ & $1.36(0.6)$ \\
$\begin{array}{l}\text { Supine } \\
\text { Active sleep }\end{array}$ & 37 & $123.2(9.4)$ & $20.99(8.0)$ & $18.19(9.2)$ & $1.32(0.4)$ \\
$\begin{array}{l}\text { Prone } \\
\text { Supine }\end{array}$ & 35 & $130.7(8.8)$ & $27.23(8.6)^{\star \star}$ & $14.87(6.5)^{\star}$ & $1.96(0.5)$ \\
\hline
\end{tabular}

${ }^{\star} \mathrm{p}<0.05 ;{ }^{\star \star} \mathrm{p}<0.001$ prone $v$ supine.

\section{Results}

HEART RATE VARIABILITY

A summary of the statistical measures for heart rate variation is given in table 1 . There were no significant differences between the mean heart rates for sleep position or sleep state. HRV, as measured by the standard deviation of R-R interval length (SD R-R), was significantly less when the babies were sleeping prone than when supine in both quiet and active sleep. The standard deviation of the amount of change from one interval to the next ( $S D \triangle R-R)$ was also significantly less in the prone position. These differences were not reflected in the aspect ratio (ratio of variance (or SD) of $\mathrm{SDR}-\mathrm{R}$ to $\mathrm{SD} \triangle \mathrm{R}-\mathrm{R})$. Poincaré plots illustrate visually this reduced variation in the decreased scatter of the points in the prone as compared to the supine position (fig 3). The plots shown are from the same individual and are each made up of about 500 consecutive R-R intervals.

All Poincaré variables had a significantly $(p<0.001)$ higher variance in active sleep than in quiet sleep.

RESPIRATORY RATE

Sleep position was not associated with changes in respiratory rate. Respiratory rates recorded in quiet sleep were: prone $34.1(10.4)$ and supine 36.2 (8.8) breaths/minute. In active sleep respiratory rates were: prone 39.9 (9.2) and supine 42.3 (9.1) breaths/minute. Significantly higher respiratory rates were recorded in active sleep than in quiet sleep in both sleep positions $(\mathrm{p}<0.05)$.

R-R INTERVAL CHANGES IN RESPONSE TO TILT A typical response of the R-R interval changes following a tilt is shown in fig 2. All infants responded to the tilt with an increase in heart rate (decrease in R-R) followed by a decrease in heart rate (increase in $\mathrm{R}-\mathrm{R}$ ) with a maximum :minimum R-R interval ratio greater than 1 . The tilt often provoked the sigh reflex. No significant differences were found between the prone and supine position on the maximum :minimum R-R interval ratio (table 2). There was a trend for the rebound bradycardia (maximum R-R) to be less in the prone sleep position but this was not significant.

\section{AROUSAL RESPONSE TO THE TILT}

Figure 4 summarises the tilt test results according to arousal code, sleep position, and sleep state. Arousability was significantly less ( $p$ $<0.01$ ) in the prone sleep position but not in quiet sleep. Arousal was much more common in active sleep in both sleep positions $(\mathrm{p}<$ 0.01 ). In quiet sleep about $72 \%$ of all tilt tests resulted in no arousal (grade 0) whereas in active sleep no arousal was noted in $30 \%$ of all tests when prone and $20 \%$ when supine. Prone positioning significantly reduced the number of full awakenings (grade 2) recorded in response to the test $(14.8 \%$ of prone tests compared with $54 \%$ supine) in active sleep.

\section{Discussion}

The main findings of this study of healthy $2-4$ month old infants sleeping prone compared 

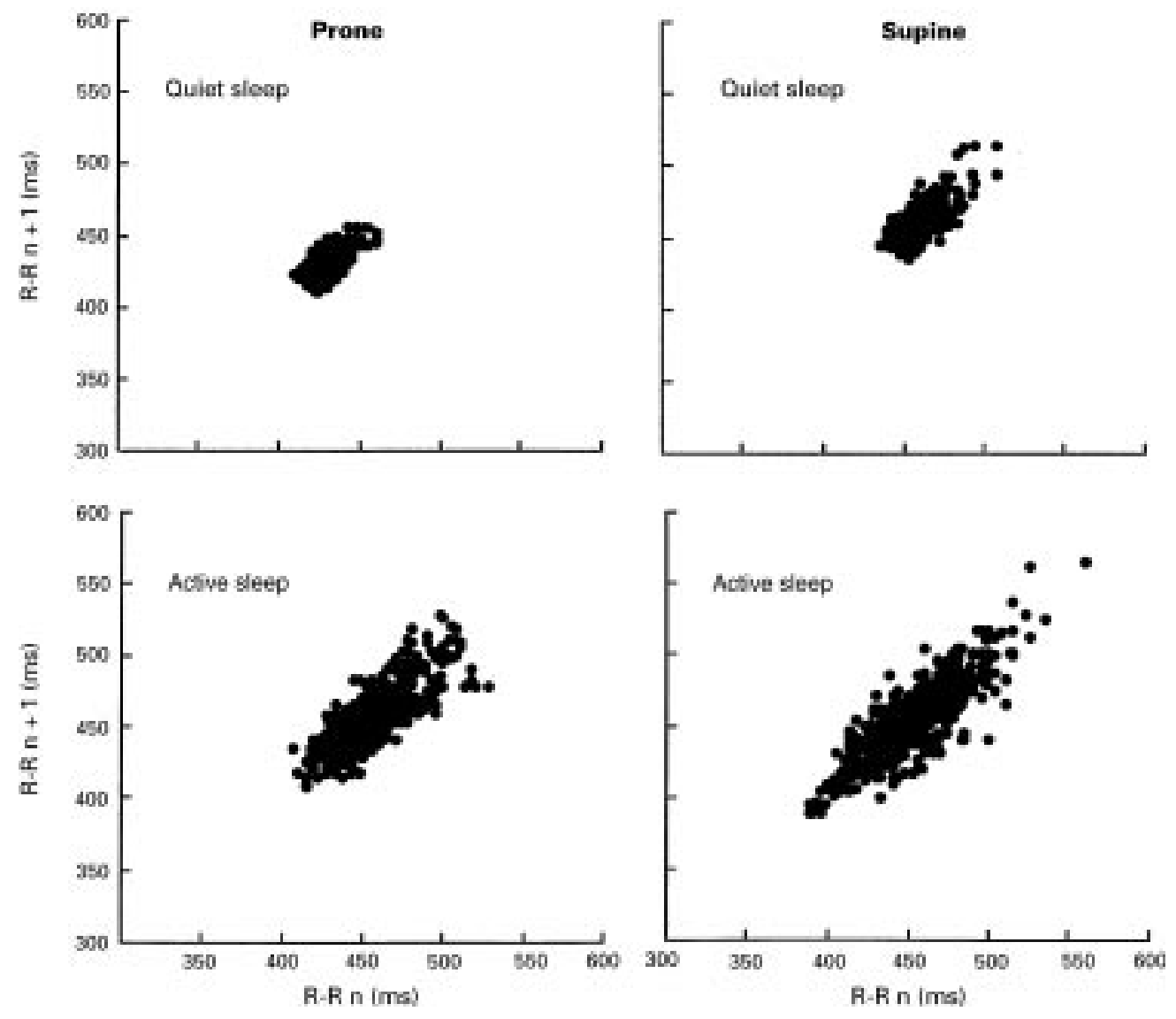

Figure 3 Example of the differences in Poincaré plots recorded from the same individual during prone and supine positioning in quiet and REM sleep.

with supine were that HRV was reduced in active and quiet sleep and arousal responses were depressed in active sleep. Physiological correlates to explain a reduction in HRV (derived from time domain and frequency domain measures) are complex. ${ }^{16}$ During rest $\mathrm{R}-\mathrm{R}$ interval lengths are constantly modified by haemodynamic, neural, and hormonal influences. It is generally accepted that a reduction in HRV can be associated with cardiac autonomic dysfunction ${ }^{17}{ }^{18}$ such as autonomic withdrawal resulting in a sympathovagal imbalance and/or high levels of sympathetic activity. ${ }^{19}$ A reduction in HRV can also result from decreased responsiveness of sinus nodal cells to neural modulation, ${ }^{20}$ and to increased respiratory rhythmicity. ${ }^{21}$

A reduction in HRV derived from Poincaré data has been reported by Schechtman et $a l^{11}$ in infants who died of SIDS, the main findings being a reduction in beat to beat point dispersions at the longer intervals (lower heart rates) in comparison to data from age matched controls. They concluded that either sympa- thetic hyperactivity, parasympathetic hypoactivity, or a combination of the two underlie these findings. As sleep position was not reported, our study suggests that some of the differences may well be related to this. A recent report examining the instantaneous variation in respiratory pattern also found a difference between SIDS babies and age matched controls, with less variation occurring in SIDS babies at slow respiratory rates. ${ }^{22}$ The confounding effect of sleep position may be important here as well.

Our finding that HRV is reduced in the prone sleep position in healthy infants confirms the findings of another group who reported a decrease in HRV in active sleep when measurements were made by spectral analyses in particular frequency bands. ${ }^{23}$ We noted a reduction in variability with the prone sleep position in both active and quiet sleep states, particularly in quiet sleep, although it also occurred in active sleep. In other words quiet sleep in the prone position produces the most

Table $2 R-R$ interval changes following a $60^{\circ}$ head-up tilt

\begin{tabular}{lllllll}
\hline & $\begin{array}{l}\text { No of } \\
\text { subjects }\end{array}$ & $\begin{array}{l}\text { Mean (SD) } \\
\text { heart rate (bpm) }\end{array}$ & $\begin{array}{l}\text { Mean }(S D) \\
\text { minimim R-R (ms) }\end{array}$ & $\begin{array}{l}\text { Mean (SD) } \\
\text { maximim R-R (ms) }\end{array}$ & $\begin{array}{l}\text { Mean (SD) } \\
\text { ratio(max:min R-R) }\end{array}$ & $\begin{array}{l}\text { Mean (SD) No of } \\
\text { beats (min-max) }\end{array}$ \\
\hline $\begin{array}{l}\text { Quiet sleep } \\
\text { Prone }\end{array}$ & 33 & $128.8(6.9)$ & $410.8(35.9)$ & $550.1(82.0)$ & $1.3(0.3)$ & $15.3(6.2)$ \\
$\begin{array}{c}\text { Supine } \\
\text { Active sleep }\end{array}$ & 34 & $125.5(8.8)$ & $414.1(28.6)$ & $585.0(82.4)$ & $1.4(0.2)$ & $15.3(5.7)$ \\
$\begin{array}{l}\text { Prone } \\
\text { Supine }\end{array}$ & 23 & $129.3(7.8)$ & $390.3(27.6)$ & $517.5(56.4)$ & $1.3(0.1)$ & $15.0(5.0)$ \\
\hline
\end{tabular}



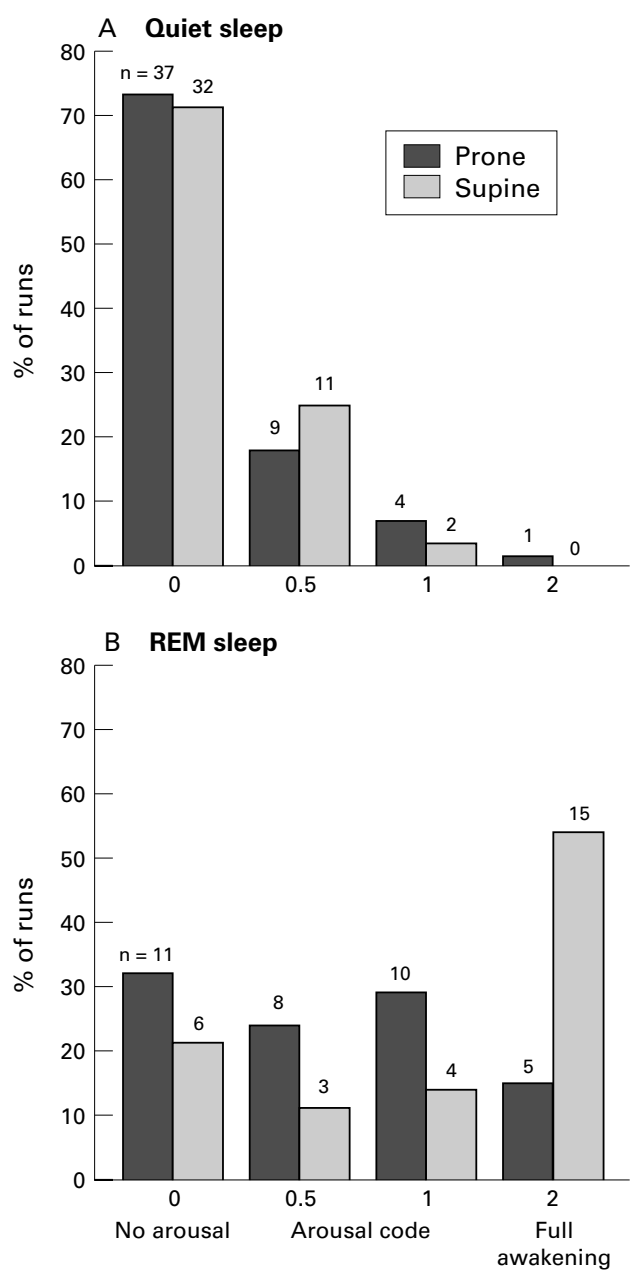

Figure 4 Frequency histograms of the arousal codes scored following a $60^{\circ}$ head-up tilt. (A) Quiet sleep in prone and supine positions; (B) REM sleep in prone and supine sleep positions. Arousal code 0: no movement at all; 0.5: startle response in tilt but remaining asleep; 1: awake with gross head movement and/or eyes open but asleep within 15 to 30 seconds and remaining so on return to horizontal; 2 : full awakening with eyes open and usually crying.

inflexible heart rate. Quiet sleep is also associated with the least chance of arousal.

A postural change between supine and prone means a positional change in the level of the carotid sinus in relation to the heart, being below the level of the heart when supine, and above when prone. Whether this has any influence on baroreceptor gain is unknown. We suggest that a reduction in "breathing effort" observed in healthy full term infants on a shift from supine to prone ${ }^{24}$ might cause a reduction in respiratory sinus arrhythmia. This could have an impact on the autonomic nervous system by dampening beat-beat variation in the prone position.

That we found no effect of sleep position on the cardiac response of infants to the tilt is perhaps not surprising, given the combination of events following the gravitational stressthat is, fall in blood pressure, transient arousals, sighs and apnoeas. Transduction of these haemodynamic and neural effects to cardiac rate must overlap so that sleep position then becomes a negligible factor. A study in anaesthetised rats of comparative changes in blood pressure (taken from direct arterial recordings) in response to a $45^{\circ}$ head-up tilt when prone and supine, reports a smaller fall in blood pressure when prone and a smaller rebound bradycardia after the tilt, ${ }^{25}$ the latter finding being consistent with the trend we found. In contrast, another study comparing blood pressure changes to a $60^{\circ}$ head-up tilt in sleeping infants reports a significantly greater fall in blood pressure (measured from automated blood pressure cuffs) when positioned prone rather than supine. ${ }^{13}$ The authors concluded that sleeping prone adversely affects autonomic function in healthy infants. Our attempts to compare blood pressure changes in infants before and after the tilt, using the same automated technique, were unsuccessful because the technique was not accurate enough, the measures could not be taken frequently enough, and inflation and deflation of the cuff often provoked arousal (unpublished findings).

$\mathrm{R}-\mathrm{R}$ interval measurements in response to the a head-up tilt (such as those used here) are most often used as a clinical tool for assessing autonomic function in adults with cardiovascular disorders. ${ }^{26}$ The test is always performed during the awake state so that the level of consciousness remains unchanged, ruling out any confounding effects of the changing sleepwake patterns we encountered. The head-up tilt has been used to identify disturbances in autonomic function of sleeping infants that have sustained an ALTE. ${ }^{27}$ In control infants (and in the infants studied here), the response to the tilt is almost always an increase followed by a decrease in heart rate. ${ }^{127} \mathrm{~A}$ significant proportion of ALTE infants responded to the tilt test by a constant increase or decrease in heart rate, a decrease followed by an increase, or no change at all. ${ }^{27}$

The tilt test proved most useful for us as a tool to assess the differences in arousal responses of infants sleeping prone compared with those sleeping supine. The finding that prone sleeping depressed arousal of infants during active sleep supports data showing the sleep promoting effects of prone positioning in similar aged infants. ${ }^{23}{ }^{28}$ Our method of scoring arousal based on observations graded from nil response to full awakening was important in terms of recognising that even slight head movement could be enough to protect the vulnerable infant from danger; the scenario being an infant who has to respond to the stress of sleeping face down into soft bedding where the simple response of head movement without full awakening may be enough to prevent rebreathing $^{29}$ or airways obstruction, ${ }^{30}$ but obviously a full awakening would be most protective. The tilt test was not a useful predictor of arousal in quiet sleep as two thirds of all tests resulted in no arousal in both sleep positions and only one test provoked a full awakening response.

In conclusion, we have shown that prone sleeping reduces the variability of heart rate in normal healthy 3 month old infants during both active and quiet sleep. Furthermore, prone sleeping reduces the ability of the infant 
to wake from test stimuli during active sleep. The findings could help explain why prone sleeping seems to be such a dangerous sleeping position even for apparently healthy babies who have no risk factors for SIDS.

We are grateful to our research nurse, Mrs Rachel Sayers, who assisted with the studies and Mrs Sheila Williams for her assistance with statistical analyses. This stud

1 Dwyer T, Ponsonby A-LB, Newman NM. Prospective cohort study of prone sleeping position and sudden infan death syndrome. Lancet 1991;337:1244-7.

2 Mitchell E, Scragg R, Stewart AW, et al. Results from the first year of the New Zealand cot death study. NZ Med $\mathcal{F}$ 1991;104:71-6.

3 Fleming PJ, Gilbert, Azaz Y, et al. Interaction between bedding and sleeping position in the sudden infant death syndrome: A population based case-control study. BMF 1990;301:85-9.

4 Tonkin SL, Partridge J, Beach D, Whiteney S. The pharyngeal effect of partial airways obstruction. Pediatrics 1979;63:261-71.

5 Kahn A, Rebuffat E, Sottiaux M, Muller MF, Bochner A, Grosswasser J. Brief airway obstruction in infants with
breath-holding spells. F Pediatr 1990;117:188-93.

6 Kemp JS, Thach BT. Sudden death in infants sleeping on polystyrene-filled cushions. N Engl F Med 1991;324:185864.

7 Bolton D, Taylor B, Campbell A, Galland B, Cresswell C. Rebreathing expired gases from bedding: a cause of cot death? Arch Dis Child 1993;69:187-90.

8 Nelson EAS, Taylor BJ, Weatherall IL. Sleeping position and infant bedding may predispose to hyperthermia and sudden infant death syndrome. Lancet 1989;i:199-201.

9 Taylor BJ, Williams SM, Mitchell EA, Ford RPK. Symptoms, sweating and reactivity of infants who die of . 7 Paediatr Child $6 ; 32: 316-32$

10 Kahn A, Van de Merckt C, Dramaix M, Magrez P, Blum D, Rebuffat E, Montauk L. Transepidermal water loss during sleep in infants at risk for sudden death. Pediatric 1987;80:245-50

11 Schechtman VL, Raetz SL, Harper RK, Garfinkel A, Wilson AJ, Southall DP, Harper RM. Dynamic analysis of cardiac $\mathrm{R}-\mathrm{R}$ Intervals in normal infants and in infants who subsequently succumbed to the sudden infant death syndrome. Pediatr Res 1992;31:606-12.

12 Fox GPP, Matthews TG. Autonomic dysfunction at different ambient temperatures in infants at risk of sudden infant death syndrome. Lancet 1989;ii: 1065-7.

13 White M, Beckett M, O'Regan M, Matthews T. Autonomic function and SIDS. Acta Paediatr 1993;389:105-6.
14 Anders T, Emde R, Parmelee AE. A manual of standardized terminology, techniques and criteria for scoring of states of sleep and wakefulness in newborn infants.. Los Ange

15 Kamen PW, Tonkin AM. Application of the poincare plot to heart rate variability; a new measure of functional status in heart failure. Aust NZ Med F 1995;25:18-26.

16 Taskforce of the European Society of Cardiology and the North American Society of Pacing and Electrophysiolog Heart rate variability. Standards of measurement, physiological interpretation, and clinical use. Circulation 1996;93:1043-65.

17 Pagani M, Lombardi F, Guzzetti S, et al. Power spectral analysis of heart rate and arterial pressure variabilities as a conscious dog. Circ Res 1986;59:178-93.

18 Dalton KJ, Dawes GS, Patrick JE. The autonomic nervous system and fetal heart rate variability. Am $\mathcal{F}$ Obstet Gynecol 1983;146:456-62.

19 Malik M, Camm AJ. Components of heart rate variability: what they really mean and what do we really measure. $A m$ 7 Cardiol 1993; 72:821-2.

20 Malliani A, Lombardi F, Pagani M. Power spectral analysis of heart rate variability: a tool to explore neural regulatory mechanisms. Br Heart 7 1994:27:1-2

21 Woo MS, Woo MA, Gozal D, Jansen MT, Keens TG, Harper RM. Heart rate variability in Congenital Central Hypoventilation Syndrome. Pediatr Res 1992;31:291-6.

22 Schechtman VL, Lee MY, Wilson AJ, Harper RM. Dynamics of respiratory patterning in normal infants and infants who subsequently died of the Sudden Infant Death Syndrome. Pediatr Res 1996;40:571-7.

23 Franco P, Pardou A, Hassid S, Kahn A. Decreased cardiac responses to auditory stimulation during prone sleep. Pediatr 1996;97:174-8.

24 Adams JA, Zabelta IA, Sackner MA. Comparison of supine and prone noninvasive measures of breathing patterns in fullterm newborns. Pediatr Pulmonol 1994;18:8-12.

25 Karakida T, Yamazaki Y, Homma S. Blood pressure and heart rate changes in streptozotocin diabetic rats, with special reference to postural hypotension. fap $\mathcal{f}$ Physiol 1991;41:589-603.

26 O'Marcaigh AS, MacLellan-Tobert SG, Porter CJ. Tilttable testing and metoprolol therapy in young patients with unexplained syncope. Pediatr 1994;93:278-83

27 Edner A, Katz-Salamon J, Lagercrantz H, Milerad J. Heart rate response profiles during head upright tilt test in infants with apparent life threatening events. Arch Dis Child 1997; 76:F27-30

28 Kahn A, Groswasser J, Sottiaux M, Rebuffat E, France P, Dramaix M. Prone or supine body position and sleep characteristics in infants. Pediatr 1993;91:1112-5.

29 Chiodini BA, Thach BT. Impaired ventilation in infants sleepin $\mathrm{g}$ facedown: potential significance for sudden infant death syndrome. F Pediatr 1993;123:686-92.

30 Waters KA, Gonzalez A, Jean C, Morielli A, Brouillette RT. The face straight-down and face-near-straight-down positions in normal, prone-sleeping infants. 7 Pediatr 1996;128:616-25. 